\title{
NUCLEAR CHRONOMETERS
}

\author{
J. J. COWAN \\ Department of Physics \& Astronomy, University of Oklahoma, Norman, OK \\ 73019, USA \\ E-mail: cowan@mail.nhn.ou.edu \\ C. SNEDEN \\ Department of Astronomy, University of Texas, Austin, TX 78712, USA \\ E-mail: chris@verdi.as.utexas.edu \\ J. W. TRURAN \\ Department of Astronomy, University of Chicago, Chicago, IL 60637, USA \\ E-mail: truran@nova.uchicago.edu

\begin{abstract}
Observations of metal-poor Galactic halo stars indicate that the abundance pattern of the (heaviest) neutron-capture elements is consistent with the scaled solar system $r$-process abundances. Utilizing the radioactive ( $r$-process) element thorium, age determinations have been made for several of these same stars, placing constraints on both Galactic and cosmological age estimates.
\end{abstract}

\section{Introduction}

Stellar observations of neutron-capture elements (i.e., those produced in the slow- or rapid-neutron capture processes) provide a number of important clues about the formation history, chemical evolution and age of these elements in the Galaxy. In particular, the abundances of the $n$-capture elements in metal-poor (i.e., low iron abundance) Galactic halo stars can be employed to determine the nature of the progenitors and the nucleosynthesis history in the early Galaxy. Further, the changes in the stellar abundance trends with respect to metallicities (and times) can provide clues about the nature of Galactic chemical evolution and the sites for neutron-capture synthesis. Just as importantly, certain long-lived radioactive elements, such as thorium and uranium, are produced entirely in the $r$-process. The abundance levels of these nuclear chronometers in the most metal-poor halo stars provide direct age determinations and hence set lower limits on Galactic and cosmological age estimates.

rprocess: submitted to World Scientific on November 4, 2018 


\section{Stellar n-Capture Abundance Distributions}

There have been a number of recent ground-based (see e.g. Sneden et al ,

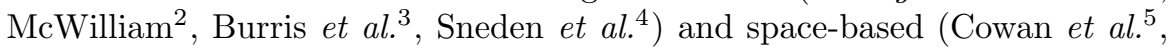
Sneden et al. . observations of metal-poor Galactic halo stars. Several of these studies have concentrated on the star CS 22892-052 (Cowan et al.:, Sneden et al. , Sneden et al. $\left.{ }^{4}\right)$. This star, while ultra-metal-poor $([\mathrm{Fe} / \mathrm{H}]=-3.1)$, is neutron-capture rich with an average abundance level of 30-40 times solar (with respect to iron) for the elements heavier than Ba. These large overabundances have allowed the detection of a number of elements for the very first time in very metal-poor halo stars. In Figure 1 (at the top) the neutroncapture abundances in CS 22892-052 for elements with atomic number, Z, $\geq 56$ are shown. Also shown in comparison is the solar system elemental $r$-process abundance distribution, indicated by a solid line. This distribution has been determined by summing the individual isotopic contributions from the $\mathrm{s}_{-}$and the $r$-process in solar system material, as determined by Käppeler et al $\mathrm{B}$ from neutron-capture cross section measurements. (See Burris et al. for more details.) It is clear in Figure 1 that there is a remarkable agreement between the elemental abundances in this star and the scaled solar system $r$ process abundances - the relative abundances of the heavy $n$-capture elements are similar in CS 22892-052 and the solar system. While this argument has been made previously, the detection of additional elements in CS 22892-052 has strengthened that argument.

While the agreement between scaled solar system $r$-process abundances and CS 22892-052 is very suggestive, it might be considered coincidental if it was a unique situation. This is not in fact the case. Recent observations of other neutron-capture rich halo stars have shown a similar pattern. The $n$-capture abundances in one such star, HD 115444, with a metallicity similar to CS 22892-052, is shown in the lower part of Figure 1 (Westin et al.e). The abundances of the elements in this star have been arbitrarily (uniformly) reduced (i.e., moved downward) for illustration purposes - the actual abundances are approximately a factor of 5 lower than in CS 22892-052. The agreement between the abundances in HD 115444 and the scaled solar system elemental $r$-process abundance curve is again excellent and extends through the heaviest stable elements including Os and $\mathrm{Pt}$ in the so-called $3^{\text {rd }} r$-process peak. Still further support for this agreement is provided by recent observations of stars in the globular cluster M15. The abundances of the heaviest neutron-capture elements in three giants in that cluster are again consistent with a scaled solar system $r$-process abundance distribution (Sneden et al. 2 ).

rprocess: submitted to World Scientific on November 4, 2018 


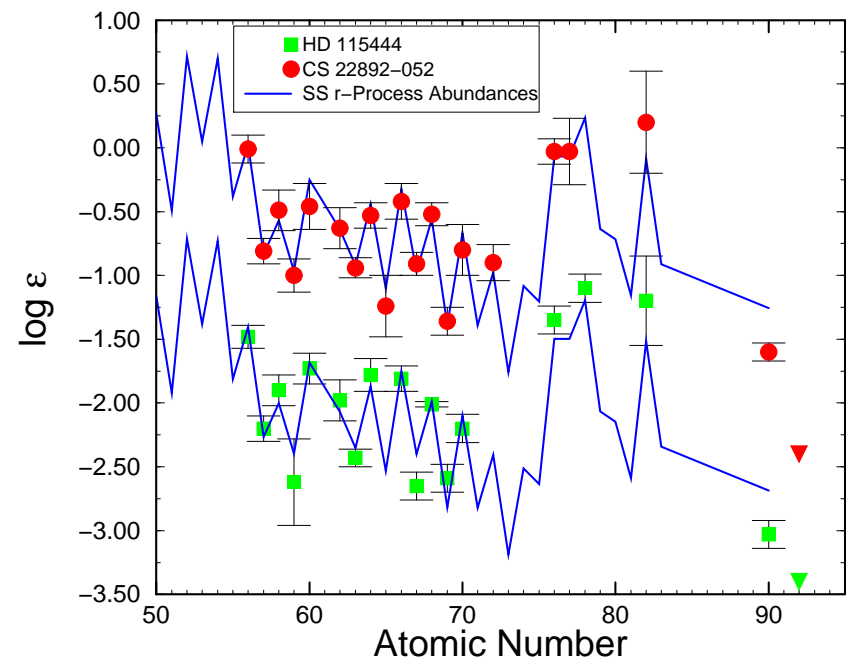

Figure 1. The heavy $n$-capture abundance patterns for the two stars CS 22892-052 and HD 115444 are compared with the solar system $r$-process abundance distribution (solid line) .

It is also apparent from Figure 1 that elements, such as barium, in CS 22892-052 and HD 115444 that are typically thought of as s-process elements (and predominantly produced by that process in solar system material - see Burris et al.3) were almost exclusively produced by the $r$-process early in the history of the Galaxy. This result affirms earlier predictions (Truran 1 ) concerning the dominance of $r$-process nucleosynthesis early in the history of the Galaxy. The detection of $r$-process material in the metal-poor and old halo stars, including the heaviest $r$-process elements, indicates an early presence of these elements in the Galaxy. That in turn also places constraints on the type of progenitors of the $r$-process elements and, in particular, suggests rapidly evolving progenitors of the halo stars. This follows since the early Galaxy appears to be chemical inhomogeneous in n-capture elements (see Sneden et al. in this volume) implying a relatively short (with respect to stellar evolutionary) timescale between the death of the progenitors and the formation of the halo stars.

Until now the element domain between $\mathrm{Zr}$ and the heavier $n$-capture elements, i.e. $40<\mathrm{Z}<56$, has been largely unexplored. For the first time Sneden et al. $\mathbf{t}^{\mathrm{t}}$ have detected six elements in this region in CS 22892-052, as 


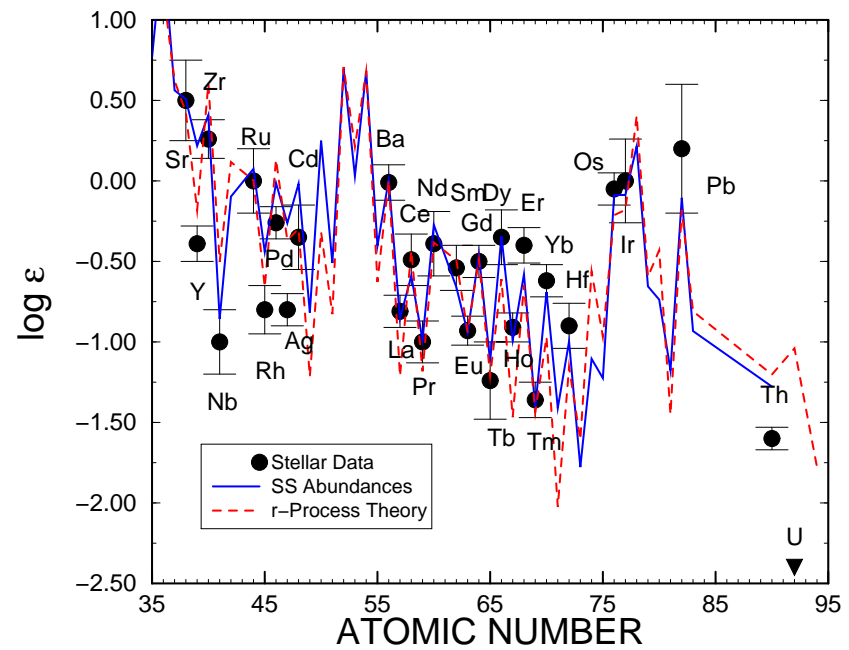

Figure 2. The total neutron-capture abundance pattern in CS 22892-052 compared with the solar system $r$-process abundances (solid line) and a theoretical $r$-process abundance curve (dashed line).

shown in Figure 2. It is clear from the abundance comparisons in Figure 2, however, that the lighter neutron-capture elements, specifically the six newly detected elements in CS 22892-052, do not fall on the same curve as the heavier neutron-capture elements. These recent observations lend support to earlier suggestions - based upon analyses of solar system meteoritic material - of a second $r$-process with the heavier elements (Ba and above) produced in more rapidly occurring events and the lighter elements in less commonly occurring syntheses (Wasserburg et al.12, Wasserburg and Qian13; see Sneden et al. in this volume for discussion.)

\section{Ages from Cosmochronometry}

The long-lived radioactive nuclei (known as chronometers) in the thoriumuranium region are formed entirely in the $r$-process and can be used to determine the ages of stars and the Galaxy. The technique of using the observed stellar thorium, particularlyas a ratio to a stable element, to obtain ages was first introduced by Butcher 14 and developed by François et al.15. The detection of thorium in CS 22892-052 and HD 115444 (see Figure 1), therefore, 
allows for chronometric stellar age estimates. The age is derived by comparing the observed stellar abundance ratio $\mathrm{Th} / \mathrm{Eu}$ with predictions of the initial (zero-decay) value of $\mathrm{Th} / \mathrm{Eu}$ at the time of the formation of these elements. (Eu is employed since it is also a predominantly an $r$-process element and has spectral features that are easily observed in most metal-poor stars.) Cowan et al. 1 and Westin et al. 0 compared the observed values of Th/Eu in CS 22892052 and HD 115444 with that in solar system material to obtain an average age of 13.8 Gyr. This estimate is in fact a lower limit on the age of these very low metallicity stars. This follows since the solar Th/Eu ratio (at the formation of the solar nebular 4.5 Gyr ago) represents a lower limit to the zero decay-age $r$-process abundances - Eu is stable and Th (although constantly produced and ejected into the interstellar medium) is partially decayed.

Further refinements in the age estimates can be obtained by determining the zero-decay abundance of thorium when produced in an $r$-process site. This, however, depends critically upon theoretical predictions for very neutron-rich nuclei, whose properties are in general not obtainable by experimental determination. We can, however, employ the stable abundances of the neutron-capture elements and the solar system $r$-process abundances to constrain the theoretical calculations and, hence, assess the reliability of any nuclear mass formulae utilized in those calculations. One such calculation, based upon the work of Pfeifer et al. 17 (see also Cowan et al. .6 for details), is shown in Figure 2 by a dashed line. It is seen in the figure that this calculation gives very good agreement for the abundance predictions of the heavy $n$-capture elements in CS 22892-052 and the solar system abundances. In this same calculation, constrained by the stable abundances, abundance predictions for the radioactive heavy elements are simultaneously derived. There is still an uncertainty associated with the abundance predictions for the radioactive chronometers thorium and uranium. Unlike the stable heavy solar system elements, such as $\mathrm{Pt}$, which can be compared directly with theoretical predictions, no such comparison is possible with Th or U. However, the $\alpha$-decay of heavier nuclei $(209<\mathrm{A}<255)$, including those from Th and $\mathrm{U}$, is responsible for the production of the stable lead and bismuth isotopes, whose abundances are known and thus can be compared with theoretical estimates. Employing this important solar system abundance constraint on the theoretical $r$-process calculations to determine the zero-decay $\mathrm{Th} / \mathrm{Eu}$ abundances, Cowan et al. 16 and Westin et al.9 found an average age for CS 22892-052 and HD 115444 of $15.6 \mathrm{Gyr}$, with an estimated uncertainty of $\simeq 4$ Gyr. Sneden et al. 19 report on thorium detections in several stars in the globular cluster M15 and obtain ages, $\simeq 14 \mathrm{Gyr}$, that are also consistent with those found for the two halo stars.

rprocess: submitted to World Scientific on November 4, 2018 
While there are still uncertainties associated with the nuclear physics predictions, this technique offers great promise for stellar dating. One large advantage is that such radioactive age determinations of very low metallicity stars are independent of, and thus avoid the inherently large uncertainties in, Galactic chemical evolution models. With the addition of more observations and better nuclear physics predictions, the current radioactive age uncertainties should be reduced. Finally, we note that while uranium has not been observed so far in either CS 22892-052 or HD 115444 (upper limits on U are indicated by inverted triangles in Figures $1 \& 2$ ), in the near future it may be possible to observe this element in some metal-poor halo stars. The addition of this other nuclear chronometer would help to constrain further age estimates for the Galaxy and the Universe.

\section{Acknowledgments}

We thank all of our colleagues who have collaborated with us on various studies of $n$-capture elements in halo stars. This research has received support from NSF grants AST-9986974 to J.J.C., AST-9987162 to C.S. and from DOE contract B341495 to J.W.T., and from the Space Telescope Science Institute grant GO-8342.

\section{References}

1. C. Sneden et al., ApJ 467, 819 (1996).

2. A. McWilliam, $A J \mathbf{1 1 5}, 1640$ (1998).

3. D. L. Burris et al., ApJ 544, 302 (2000).

4. C. Sneden et al., ApJ 533, L139 (2000).

5. J. J. Cowan et al., ApJ 460, L115 (1996).

6. C. Sneden et al., ApJ 496, 235 (1998).

7. J. J. Cowan et al., ApJ 439, L51 (1995).

8. F. Käppeler et al., Rep. Prog. Phys. 52, 945 (1989).

9. J. Westin et al., ApJ 530, 783 (2000).

10. C. Sneden et al., ApJ 536, L85 (2000).

11. J. W. Truran, $A \& A$ 97, 391 (1981).

12. G. J. Wasserburg et al., ApJ 466, L109 (1996).

13. G. J. Wasserburg and Y.-Z. Qian, ApJ 529, L21 (2000).

14. H. R. Butcher, Nature 328, 127 (1987).

15. P. François et al., A $6 A$ 274, 821 (1993).

16. J. J. Cowan et al., ApJ 521, 194 (1999).

17. B. Pfeiffer et al., Z. Phys. A 357, 235 (1997). 